\title{
Conceptual analysis and empirical research in medical philosophy and medical ethics
}

\author{
Wim Dekkers • Bert Gordijn
}

Published online: 15 October 2009

(C) Springer Science+Business Media B.V. 2009

One cannot think of medical practice and medical science without being aware of the phenomena of health and disease (or illness). Since time immemorial health and disease are essential concepts in the philosophy of medicine and health care. It is a long ongoing debate. The concepts of health and disease have been studied and analyzed for decades without reaching any consensus on their content. In the literature, it is argued that the concept of disease is complex, vague, slippery, indefinable, or even dead. In the first paper in this issue, Bjørn Hofmann argues that the arguments for the vagueness, complexity and indefinability of the concept of disease are not overly convincing and that the claim that the concept of disease can be abandoned altogether needs better arguments. His conclusion is that it appears at least as hard to show that disease is indefinable as it is to define it. Marianne Boenink also embarks on the philosophy of disease, especially on the relationship between the emergence of new technologies such as molecular medicine and concepts of disease. It is widely acknowledged that new technologies often not only produce new 'ontologies', but also new roles and new ethical responsibilities. The technological constitution of disease raises extensive philosophical debates, but ethical analyses of new biomedical technologies only rarely include conceptual clarification of disease concepts implied by these new technologies. The author argues that it is useful to start with an analysis of concepts of disease which are implied in new emerging technologies, when anticipating ethical issues of biomedical technologies. Thus, a conceptual analysis should precede the ethical debate.

W. Dekkers $(\bowtie) \cdot$ B. Gordijn

UMC St Radboud Nijmegen, 114 IQ Healthcare, Section Ethics, PO Box 9101, 6500 HB Nijmegen, The Netherlands

e-mail: v.hulsman@iq.umcn.nl
The third article in this issue also deals with a conceptual problem. The policy responses to suicide and schizophrenia in the UK - and in many other countries - are predicated on notions of global irrationality as a justification for paternalistic interventions. Jeanette Hewitt argues that it is theoretically possible that suicide may not be an irrational response to the suffering experienced by people with a severe and enduring mental illness and that suicide is not necessarily a consequence of their mental illness per se. What has been conceptualized as "psychopathological" by psychiatric perspectives may be a normal reaction of hopelessness to a realistic appraisal of the course and consequences of living with schizophrenia. However, this point of view does not preclude intervention of any kind to prevent suicide. Also the fourth article in this issue is analytic and argumentative in nature. Dan Egonsson comes up with a new interpretation of the Substituted Judgement Standard (SJS). SJS is commonly understood in a counterfactual and purely hypothetical way culminating in the question what decision the patient here and now would have made, had he or she been competent. Egonsson believes there is another reading in which the emphasis is more on the past, in so far that it bears on the present situation, than on the now. He proposes an alternative, factual, interpretation of the SJS in which the surrogate is required to infer what the patient in the past actually thought about a particular treatment that is being considered.

A few decades ago, medical ethics moved from a predominantly theoretical discipline to a discipline that not only explicitly reflects on empirical findings, but also considers empirical research as an important part of its endeavour. The next two articles are in line with this empirical turn in bioethics. Yvonne Denier et al. report about the findings of an empirical, qualitative study based on in-depth interviews with 18 nurses from Flanders 
(Belgium). These nurses were interviewed about their experiences with the care for patients who had an actual request for euthanasia. All nurses described their involvement in the euthanasia procedure as a grave and difficult process, not only on an organizational and practical level, but also on an emotional level. "Intense" appeared to be the dominant feeling among the nurses. They experience a kind of discrepancy: euthanasia might be a nice death that happens in dignity and with respect, but it is an unnatural death. Roxana Behruzi et al. also report about an empirical study albeit it in an entirely different field. The goal of their article is to identify the professionals' perception of the potential obstacles and facilitating factors for the implementation of "humanized" care in high risk pregnancies. They interviewed 21 midwives, obstetricians and health administrators in nine different Japanese institutions. Based on their empirical findings they arrive at some suggestions that could improve obstetric care in high risk pregnancies.

While the previous two papers reported about empirical findings, the next two deal with methodological problems related to empirical research in bioethics. Niels Lynøe et al. are interested in the question how to reveal disguised paternalism. Physicians, at least in a Swedish setting, are unlikely to give explicitly paternalistic reasons when they are asked about their attitudes toward patients' involvement in decision-making. There is a considerable risk that they will give socially correct answers. Lynøe et al. suggest that disguised paternalism can be revealed with the help of indexes based on certain responses in postal questionnaires. Questionnaires exploring paternalism might be improved by including not only general statements, but also casespecific questions. In their article they give concrete examples of such specific questions. In her paper, Leah McClimans suggests a new approach to quality of life research. She critically examines two main orientations in standard health-related quality of life measurement, that is standardized and individualized measures. These measures are considered attempts to stimulate patient-centered health care and to provide for patient self-determination, but fail to do so. A so-called dialogic approach to quality of life research primarily requires researchers to learn how to continue asking questions about how they might best understand a specific measure's outcome.

The final paper in the category 'Scientific contribution' is a report "from the bioethical field". Pierre Mallia describes how Malta, a traditional Roman Catholic Society, struggles with new reproductive technologies such as in vitro fertilization (IVF). Recently the Maltese government proposed to legislate human reproductive technologies, in particular IVF which has been offered privately and practiced for over two decades without controlling legislation. However, due to its political sensitivity, legislation has been postponed. Mallia's article is an attempt to illustrate the difficulties encountered focusing on the role of the Catholic Church, the government, and the media. His article is also a tentative step in the direction of a further dialogue by formulating a couple of recommendations.

Finally, this is the time and place to announce that Wim Dekkers is going to end his activities as editor-in-chief. Wim Dekkers began his work for Medicine, Health Care and Philosophy as managing editor at the start of the journal in 1998. In 2004 he replaced the former editor-inchief, Henk ten Have, and did in fact most of the editorial work. As of 2005, volume 8, Wim Dekkers and Bert Gordijn have jointly been editors-in-chief. We are happy to announce that, as of 2010, Henk ten Have will again pick up the job of being one of the two editors-in-chief. 